\title{
La investigación formativa en Educación Superior: modelo para docentes y estudiantes
}

\section{Formative research in Higher Education: a model for teachers and students}

\author{
Bolívar Cabrera Berrezueta $^{1 *}$, Nancy Cárdenas Corder $^{1}$ o y Darwin Gabriel García Herrera ${ }^{1}$ \\ Universidad Católica de Cuenca, Ecuador \\ *bolivarcabrera@ucacue.edu.ec
}

DOI: https://doi.org/10.26871/killkanasocial.v4i2.612

\begin{abstract}
Resumen
El presente artículo aborda temas que se relacionan con la investigación formativa en la Educación Superior, mediante la relación de los ejes sustantivos como: docencia, investigación y vinculación con la sociedad. Estas funciones van encaminadas a establecer un modelo para los docentes y los estudiantes con la finalidad de desarrollar competencias investigativas a través de una adecuada fundamentación epistemológica y filosófica, con el uso de metodologías activas y el aprovechamiento de las tecnologías emergentes. La metodología empleada es de tipo bibliográfica descriptiva. Para el modelo propuesto se utilizó la modelación como método de investigación. Finalmente, la calidad de la educación exige docentes y estudiantes investigadores con nuevos retos y desafíos, lo cual implica el desarrollo del pensamiento crítico, reflexivo e innovador de forma que les permita ser gestores en la búsqueda de problemas educativos que enfrenta la sociedad y la capacidad de brindarles la solución oportuna en bien del país.
\end{abstract}

Palabras clave: Epistemología, metodologías activas, aprovechamiento de las tecnologías, modelo.

\begin{abstract}
This article addresses topics related to the Higher Education Formative Research, through the relationship of the fundamental axes such as teaching, research, and links with the society. These functions aim the establishment of a model for teachers and students to develop research skills and appropriate Epistemological and Philosophical foundation, by using active methodologies and taking advantage of the emerging technology. The methodology used is of descriptive bibliographic type. The research method used for the proposed model was modelling. Finally, good quality of education requires research teachers and students, with new challenges, which implies critical, reflective and innovative thinking development. These characteristics will allow them to become managers in searching for educational problems facing society and the ability to provide timely solutions to benefit the country.
\end{abstract}

Keywords: Epistemology, active methodologies, use of technologies, model.

\section{Introducción}

En la actualidad la sociedad, exige cambios que fomenten la calidad de los procesos educativos, evidenciándose la necesidad de fortalecer la formación investigativa con fundamentaciones y argumentos que permitan el desarrollo de competencias orientadas hacia aprendizajes con pensamiento crítico y reflexivo con la articulación de las funciones de la docencia, investigación e innovación y vinculación con la sociedad.

Velandia, Serrano y Martín (2017) mencionan que formación investigativa en educación superior es de suma importancia para la articulación pedagógica del quehacer universitario y la práctica profesional implícita en cada una de las carreras.
Estudios realizados por Tapia, Ledo y Estrabao (2017), Navarrete (2017) Hurtado y Rodríguez (2016), coinciden en que la formación investigativa es el pilar fundamental en la formación universitaria ya que tanto los estudiantes como los docentes deben confluir en una misma sintonía para desarrollar investigaciones de tipo formativas y científicas que permita el desarrollo de competencias profesionales, basadas en indagaciones constantes que apunten a una mejor continua.

Para Portela, Ortega y Amaro (2018) la investigación disciplinar es la base para la mejora continua de la calidad educativa superior, lo que implica el involucramiento de docentes y estudiantes en el escenario donde se desarrolla 
el aprendizaje, donde el componente investigativo es la base de la formación del profesional.

Las líneas teóricas del presente artículo son: abordaje epistemológico para la investigación, metodologías activas, aprovechamiento de los recursos tecnológicos emergentes. En lo que corresponde al abordaje epistemológico se concibe como teoría, tratados, crítica y filosofía de la ciencia, de las metodologías activas descritas se orienta hacia los aprendizajes basados en problemas, proyectos, estudio de casos y trabajo cooperativo, mismos, que permiten el desarrollo de competencias investigativas basadas en el aprovechamiento de recursos tecnológicos, flexibles, integradores y contextualizados.

La particularidad de este artículo es la integración disciplinar de la Filosofía, Currículo y Tecnología Educativa. Se complementa con la presentación de un modelo de investigación formativa para docentes y estudiantes de Educación Superior.

Realizada la investigación bibliográfica no se evidencia trabajos similares con un enfoque interdisciplinar, que permita profundizar en el objeto investigado, la aplicación de metodologías activas acordes a este nuevo proceso de indagación y la superación del uso de la tecnología, por una nueva concepción filosófica del aprovechamiento de la tecnología.

\section{Metodología}

La metodología empleada tiene un enfoque bibliográfico descriptivo, mismo que permite recopilar la información teórica y puntualizar aspectos relevantes de diferentes aportes de investigadores. El alcance de la investigación integra el objeto indagado, a partir de la fundamentación epistemológica, la identificación de regularidades de las metodologías activas y el desarrollo de competencias investigativas.

Para la elaboración del modelo propuesto se utilizó la modelación como método de investigación que permite la secuenciación del proceso indagativo, desde la contraposición necesidad-realidad, hasta los aportes a la teoría y a la práctica del proyecto investigado.

\section{Abordaje epistemológico para la investigación}

A lo largo de la historia de la humanidad aparecen diferentes filósofos e investigadores que proporcionan variadas explicaciones y juicios en torno a los problemas que son intrínsecos con el ser humano, así como de todo aquello que le rodea y se relaciona en busca de la verdad y nuevos conocimientos.

De los aprendizajes en las aulas de clase, de variadas lecturas realizadas e inquietudes de la problematicidad de la existencia se puede afirmar que el hombre de la edad antigua centraba su admiración en lo divino, sobrenatural y de la edad media por definir los grandes enigmas de su vida. El tema del conocimiento en estas etapas, fue un problema secundario, dándose importancia a lo divino y luego al hombre. El personaje Hamlet de Shakespeare (1998) al referirse al hombre expresa:

¡Qué admirable fábrica es la del hombre! ¡Qué noble su razón! ¡Qué infinitas sus facultades!” ¡Que expresivo y maravilloso en su forma y sus movimientos! ¿Qué semejante a un ángel en sus acciones! Y en su espíritu ique semejante a Dios! Él es sin duda lo más hermoso de la Tierra, el más perfecto de todos los animales. (p. 61)

Expresión del personaje Hamlet que evidencia la reivindicación del hombre sobre la concepción teológica y panteísta.

Descartes, padre de la filosofía moderna, acentúa el papel de la razón en la adquisición del conocimiento. $\mathrm{Su}$ pensamiento, parte desde la duda, especialmente de los sentidos y surge la primera certeza, al que denomina "cogito ergo sum" (pienso, luego existo).

Para fundamentar el conocimiento, Descartes (1968), parte del hecho que la verdad no puede ser puesta en duda y proclama la duda como método. Los cuatro preceptos (partes) del método son:

Primero, no aceptar nunca cosa alguna como verdadera que no conociese evidentemente como tal". El segundo, dividir cada una de las dificultades que examinase en tantas partes como fuera posible y como se requiriese para su mejor resolución. El tercero, conducir ordenadamente mis pensamientos. Y el último, hacer en todas partes enumeraciones tan completas y revistas tan generales que estuviese seguro de no omitir nada. (p. 65)

Más tarde Kant coloca en el centro de su filosofía al sujeto, desplazando al objeto de conocimiento. Este hecho conllevó a la denominación de giro copernicano, aunque Humberto Piñera Llera denomina la inversión copernicana, en tanto "que, si hasta ahora se aceptaba que el pensamiento se adapta a las cosas, Kant entiende que son las cosas las que se adaptan al pensamiento" (Piñera, 1959, p. 209).

La filosofía Kantiana se desarrolla a partir de cuatro interrogantes: “1) ¿Qué puedo saber? 2) ¿Qué debo hacer? 3) ¿Qué puedo esperar? 4) ¿Qué es el hombre?” (GonzálezPérez, 2015, p. 420). A la primera pregunta responde con base en la argumentación de su metafísica para llegar al conocimiento con base a la formulación de diferentes preguntas, tales como ¿qué conocemos?, ¿cómo conocemos?

Ortega y Gasset (1957) involucra al hombre con el mundo, con el contexto social en el que se desenvuelve para identificar sus problemas, necesidades, requerimientos en pos de contribuir en las soluciones: "El destino del hombre es, pues, primeramente, acción. No vivimos para pensar, sino al revés: pensamos para lograr pervivir" (43). Aseveración que constituye advertencia que el pensamiento es cualidad primaria y fundamentalmente acción del hombre.

Las sistematizaciones realizadas en torno al conocimiento de diferentes filósofos o pensadores en el recorrer de la historia, permite colegir que el conocimiento es acción propia del ser humano, para alcanzar el discernimiento, la 
fundamentación del conocimiento humano y saber qué son las cosas.

Este epígrafe centra el enfoque entorno a lo que es la epistemología: proviene de las palabras griegas "episteme, ciencia y logos, tratado, epistemología significa tratado de la ciencia" (Presta, 2015, p. 327). Viene a ser la teoría, el tratado y crítica de la ciencia, la filosofía de la ciencia. Sinónimos de epistemología son: crítica del conocimiento, gnoseología, teoría del conocimiento. Además, el objeto de la epistemología "es el conocimiento humano en sus relaciones con el mundo real" (Enciclopedia Universal Ilustrada Europeo Americana, 1930, p. 2297).

El conocimiento como objeto del conocimiento, se puede denominar metaconocimiento. Al respecto, Morín, el autor de la teoría de la complejidad expresa: "Aquí, podemos esbozar el punto de vista epistemológico que permite controlar, es decir, criticar, trascender y reflexionar sobre nuestra teoría" (Morin y Pakman, 1990, p. 71) en referencia a la crítica del conocimiento.

Morín en su tratamiento del tema coherencia y apertura epistemológica se refiere a la relación sujeto-objeto y también establece el vínculo entre el investigador y el objeto de su conocimiento.

La relación investigadora - objeto conlleva al investigador a orientar el enfoque del artículo en la fundamentación epistemológica de la investigación formativa desarrollada por docentes y estudiantes como aquella que fusiona los procedimientos de investigación, exploración y organización del conocimiento.

Sin embargo, existen diferentes abordajes respecto a la fundamentación epistemológica, desde que la "ciencia en el debate contemporáneo tiene que ser retomada como categoría central de análisis en el campo epistemológico" (Presta, 2015 , p. 93) como un pensar epistemológico universitario dentro de la realidad social.

El requerimiento que los investigadores tengan un cambio de actitud y no deben limitarse a reproducir propuestas de otros autores (Zeña, 2015), como insinuación a la incorporación de la fundamentación epistemológica para alcanzar la verdad, en relación armónica teoría-práctica.

Los problemas epistemológicos de la "cientificidad y métodos" (Navarrete, 2017, p. 232) que presentan las investigaciones en ciencias de educación en busca de nuevas alternativas académicas en precisar el conocer acerca del objeto investigado.

La epistemología de acuerdo a (Vildósola, 2017) refiere como una propuesta multidimensional que parte del conocimiento epistemológico que tiene como centro el desarrollo de actividades basadas en una visión metodológica activa, que promuevan cambios en la relación investigadorobjeto de estudio.

Tapia, Ledo y Estrabao (2017) caracterizan los fundamentos epistemológicos de la investigación formativa como una "estrategia pedagógica que permite acceder a los contenidos y formas culturales, a la vez que el proceso de socialización configura su propia identidad personal única e irrepetible en la medida que se profundiza en la construcción del conocimiento" (p. 11).

Los enfoques hasta ahora presentados, conlleva a encontrar como regularidades que la epistemología constituye la base o fundamento para la construcción del conocimiento. Como aporte del investigador a esta teoría, se parte de la premisa del cambio paradigmático, que el conocimiento se encuentra en constante construcción, nuevas verdades se indagan y se descubren.

El conocimiento parte por identificar los problemas del contexto declarados en los planes nacionales de desarrollo, zonales o regionales, provinciales y locales, declarados como información primaria y la específica identificada por los investigadores en acción inter y transdisciplinar, con la participación conjunta de docentes y estudiantes, para aprehender la realidad.

La epistemología, como filosofía de la ciencia, es el componente que sustenta la ruta de la investigación: las situaciones problemáticas del contexto, contraponer la necesidad a la realidad, el objeto investigado, campo de acción, objetivos, metodología, abordados con base a los dominios o fortalezas en lo académico, aportes de los debates académicos generados a nivel de disciplinas, subdisciplinas, grupos de apoyo pedagógico en aras de proporcionar aporte a la teoría y a la práctica, orientada a la mejora de la calidad, como concepto no concluido, sino en construcción.

Esta fundamentación epistemológica de la investigación formativa es apertura al tratamiento de metodologías activas que conllevan a alcanzar conocimientos o aprendizajes relevantes, así como, no del uso, sino del aprovechamiento de las tecnologías de información y comunicación en el ámbito educativo.

\section{Metodologías activas en investigación}

Las metodologías activas constituyen un aporte para desarrollar competencias en la investigación, en donde están involucrados los docentes y los estudiantes de Educación Superior, entre las diferentes metodologías se considerarán las siguientes: el Aprendizaje basado en problemas, el Aprendizaje Basado en Proyectos, el Estudio de Casos y el Colaborativo; en cuanto al Aprendizaje Basado en Problemas (ABP), según: Paredes (2016) se fundamenta en el modelo del constructivismo que centraliza su quehacer en el estudiante, para que sea el actor de su propio aprendizaje y a decir de Gómez (2010) es un procedimiento dinámico de enseñanza-aprendizaje que se desempeña mediante alternativas para la solución de los problemas vinculados a través de la mediación entre el individuo y el contexto; es así, que lo central del aprendizaje basado en problemas reside en descubrir, puntualizar, investigar y solventar los problemas y esto se alcanza con la relación entre el docente, el aprendizaje y el estudiante.

El Aprendizaje Basado en Problemas, crea en el docente y en el estudiante la responsabilidad de su autoaprendizaje, le permite hacer un diagnóstico para identificar la 
problemática; además, le ayuda a razonar de forma científica planteando una hipótesis para llegar a descubrir de manera cronológica la solución del problema, le apoya a realizar un trabajo colaborativo con sus compañeros mediante una buena comunicación, la adquisición de conocimientos y la capacidad de autoevaluación. Trabajar con esta metodología es factible; puesto que, permite indagar, comprender, asimilar y aplicar nuevos conocimientos para dar solución a un problema que se pretende investigar; de esta forma, los estudiantes actúan como los autores de su aprendizaje, proporcionando en el docente el papel de mediador.

La investigación en Educación Superior, amplía los conocimientos tanto de docentes como de los estudiantes con el fin de desarrollar competencias investigativas, que les permitan generar el hábito en la lectura y en la escritura. Leer para investigar mediante el acceso a los apoyos tecnológicos y a herramientas en las diferentes bases científicas a través de las páginas web y los respectivos enlaces para acceder a varias revistas como contacto para que sirvan de guía en la escritura de artículos científicos. Investigar implica un compromiso para la generación de nuevos conocimientos, lo que, involucra a docentes y a estudiantes a la práctica investigativa dentro y fuera del proceso educativo.

Continuando, con la metodología del aprendizaje basado en proyectos, se le define como: un método que apoya al proceso de la investigación con el propósito de dar respuesta a un problema abstracto con el objetivo de brindar soluciones al problema encontrado; puesto, que esto, da la oportunidad de generar nuevos conocimientos (Ramírez y Santana, 2014).

En tanto, la metodología de enseñanza - aprendizaje ordenado que facilita a los estudiantes obtener conocimientos y destrezas mediante un procedimiento de indagación organizado basado en argumentos complejos y verídicos que se originan en labores y servicios (Reibán, Rodriguez, y Zeballos, 2017), el aporte de los autores permite conocer que la metodología del aprendizaje basado en proyectos, acompaña en el proceso de enseñanza aprendizaje que se fundamenta en tareas, es un procedimiento colaborativo entre los estudiantes, fomenta el aprendizaje individual y el autónomo en donde tienen la responsabilidad de construir su propio aprendizaje a través de la investigación para llegar a descubrir problemas para luego plantearlos en proyectos.

Los proyectos en la actualidad, se han convertido como una de las formas más idóneas para aplicarlo en el aula de clase; puesto, que logra en los estudiantes la investigación, esto, surge de varias interrogaciones que son oportunas por parte de los estudiantes. Con la ejecución de un proyecto se indaga y se expresa necesidades, de ahí nace el interés para la formulación de preguntas que establecen en su trabajo, investigan nuevos conocimientos para enriquecerse y buscar los resultados y por ende presentar sus propuestas.

Travieso y Ortiz (Travieso y Ortiz, 2018) menciona que el aprendizaje por proyectos apoya el conocimiento de forma general y relacional. Partiendo desde este punto de vista, los proyectos proveen el procedimiento de la información que vaya a ser pertinente a cada estudiante y que de esta forma le adecúa a instituir sus propias vinculaciones entre los diversos temas, basado a problemas o hipótesis, ello, le ayuda a la cimentación de su propio aprendizaje y fortalecer la investigación.

Otra de las metodologías activas que apoyan en la investigación es el estudio de casos, puesto, que les apoya a los estudiantes analizar diversas situaciones profesionales que son presentadas por el docente en el proceso de enseñanza aprendizaje con el propósito de lograr obtener soluciones a los problemas que son cotidianos en la sociedad. Es imperioso que el docente aplique esta metodología en su proceso educativo mediante la discusión de un tema con el propósito de fomentar la investigación y para ello, se puede analizar un caso, esto, permitirá evidenciar el logro alcanzado en los aprendizajes.

El estudio de casos, tiene como objeto la comprensión de las dinámicas que se encuentran en el contexto relacionado con diversos métodos para la recolección de evidencias cualitativa y cuantitativa (González-Pérez, 2015). El docente es el indicado para diseñar y adaptar el estudio de caso de acuerdo a la formación del estudiante y reconociendo sus características biopsicosociales, los contenidos tratados en el aula de clase deben dar respuesta al objetivo investigado. El estudiante se asocia a una situación basado en su experiencia para recurrir al análisis respectivo. El propósito de dicha situación es involucrar un problema que se constituye en la oportunidad de enfrentar retos que provocará en el estudiante la toma de decisiones.

En la metodología, estudio de casos, el docente interviene como coordinador y está al frente de los diferentes casos presentados por los equipos de trabajo provocando la discusión, la participación y dando sus criterios acerca de la resolución del caso (De la Fe Rodríguez, VidaurretaPorrero, Gómez, y Corral, 2015).

Es importante explicar que a pesar de que los estudiantes estén trabajando en equipos la ejecución del trabajo, apoya al docente en la obtención de los argumentos necesarios para evaluar de forma individual y adquirir las competencias de los estudiantes para la toma de decisiones. De esta forma y como un refuerzo los estudiantes elaboran preguntas de retroalimentación para fortalecer a todo el grupo y prepararlos para nuevos conocimientos.

La metodología del trabajo colaborativo implica que los equipos participen con las tareas y los criterios para un logro común; es decir, es un procedimiento donde cada estudiante refuerza su conocimiento de forma más amplia; es así, que las tareas en equipo resulta más fructífero que hacerlo solo. A decir de Vildósola (2017) fomentar el trabajo colaborativo apoya para potenciar la creatividad e innovación de los estudiantes a través de sus aportes y criterios y con ellos, llegar a un acuerdo común mediante la participación mutua y compartida para mejores resultados.

El trabajo en equipo docente coordinando metodología, recursos y acciones y trabajo en equipo discentes 
quienes para aproximar lo máximo posible la actividad práctica a la realidad de gestión escolar se constituye en equipos directivos, asumiendo roles de gestión y dirección de los centros virtuales (Álvarez, Grau, y Pertegal, 2016, p. 74).

El aprendizaje que guíe a la investigación tanto para docentes y estudiantes, se basa en el trabajo colaborativo para desarrollar competencias mediante el trabajo en equipo; pero, para esto es imperioso que el docente conforme los equipos de trabajo de forma adecuada y motivar a los estudiantes hacia la cooperación para que todos los miembros del grupo trabajen con responsabilidad y de esta manera identifique el problema para que sea investigado y se busque soluciones oportunas.

Cada una de las metodologías analizadas fomenta a nuevos conocimientos y propenden a la investigación como el eje sustantivo que proyecta a una educación de calidad y responde a las exigencias de Educación Superior.

\section{Desarrollo de competencias investigativas a partir del aprovechamiento de las tecnologías}

El vertiginoso desarrollo científico y tecnológico actual, así como su propia incertidumbre, han incidido directamente en diversas áreas del conocimiento, propiciado que sus procesos investigativos adquieran nuevos matices y una mayor importancia en la sociedad contemporánea, Cárdenas, Guerra y Soler (2017) mencionan que el aprovechamiento de las tecnologías emergentes permiten el desarrollo y la adquisición de competencias investigativas, mismas que de acuerdo con Reibán, Rodríguez y Zeballos (2017) son de vital importancia para una óptima formación investigativa.

La formación investigativa, en el ámbito universitario actualmente se enfrenta con una nueva disyuntiva la cual gira en torno al ¿cómo desarrollar competencias investigativas en su profesorado y estudiantes? respuesta que de acuerdo con Espinoza, Rivera y Tinoco (2016) puede ser dada mediante un modelo que permita y propicie la integración de conocimientos, saberes y actitudes en la búsqueda de una innovación social y el aprovechamiento del uso de las Tecnologías de la Información y Comunicación (TIC) para generar investigación.

Desde esta perspectiva es fundamental en primera instancia, apropiarnos de la concepción misma de una competencia, la cual según Baranda (2005) constituye "un sistema de conocimientos, habilidades, valores y cualidades de la personalidad que se movilizan en función de las necesidades individuales y sociales, así como de los motivos, intereses y actitudes del profesional" (p.74) mismo que a su vez permite un desempeño óptimo de su profesión.

Esta concepción pedagógica permite aseverar que el músculo impulsor en la construcción de aprendizajes significativos y el desarrollo de competencias son los motivos, intereses, necesidades y actitudes de cada individuo.

En este sentido el aprovechamiento de las habilidades tecnológicas innatas en la sociedad actual e intere- ses propios de cada persona, propiciará el desarrollo de competencias en el ámbito investigativo, siempre y cuando no se pierda la perspectiva horizontal del planteamiento pregunta-respuesta, que se suscita en el argot académico y profesional.

En este sentido, el aprovechamiento de uso de tecnologías en el desarrollo de competencias investigativas adquiere valor y confluye en la manera sistémica y armónica para la búsqueda de información científica, selección de publicaciones relevantes y la organización de la información, para a su vez promover el trabajo colaborativo y cooperado a través de la construcción de comunidades académicas, científicas e investigativas.

En la misma línea Benito y Salinas (2016) establecen una interdependencia entre los recursos infotecnológicos existentes, los requerimientos de un proceso de investigación científica y la construcción de nuevos conocimientos científicos que el individuo adquiere con la práctica progresiva y la realización de investigación formativa.

En base a las premisas antes mencionadas y en concordancia con Cárdenas, Guerra y Soler (2017) el desarrollo de competencias investigativas mediadas por el uso de tecnologías debe poseer las siguientes características:

Flexible: Puesto que debe permitir la posibilidad de adaptación permanente a los cambios y necesidades individuales y sociales.

Integradora: Ya que debe facilitar la ejecución de un proceso dinámico y multifactorial que posibilite el análisis y la integración de los criterios y evidencias de desempeño investigativo en diferentes áreas del conocimiento.

Contextuada: Porque se debe ser ejecutada en diferentes situaciones y procedimientos en dependencia del actuar y a su vez permita la unificación de las necesidades de cada persona o contexto en concreto

\section{Modelo de investigación formativa para docentes y estudiantes}

Según Shakespeare y Hamlet (1998) la modelación es la reproducción de las propiedades del objeto que se investiga en otro semejante que se construye según determinas normas o reglas. Este objeto semejante o análogo se denomina modelo.

Todo modelo debe sustentarse en principios. Para la construcción del modelo de investigación formativa se asume los principios declarados por Boff (2002), que son: deducción por analogía, consistencia lógica, carácter sistémico, simplicidad y asequibilidad.

Principios que establecen semejanzas entre los componentes del objeto real y el modelo de investigación formativa propuesto (Deducción por analogía); con la respectiva fundamentación científica de sus elementos o solidez del modelo (Consistencia lógica); relaciones del docente y estudiante con los problemas, necesidades del contexto $(\mathrm{Ca}-$ rácter sistémico); Asequible, sin perder valor y el carácter científico (Simplicidad y asequibilidad). 
De otra parte, Cotino (2012) presenta características fundamentales en el ámbito de los derechos sociales, que las denomina como las cuatro A: "Asequibilidad, Accesibilidad, Adaptabilidad, Aceptabilidad", que el investigador las asocia al modelo de investigación formativa:

- Asequibilidad hace referencia a lo factible de la ejecución del modelo, en tanto que relaciona la necesidad con las situaciones problemáticas, así como hacia la solución de problema, a los aportes a la teoría y a la práctica.

- Accesibilidad porque el modelo es comprensible y está al alcance de todos (docentes y estudiantes) para la gestión investigativa.

- Aceptabilidad por cuanto que el modelo responde a criterios de significatividad, relevancia y calidad como un concepto inacabado y en constante construcción.

- Adaptabilidad o flexibilidad hace referencia a la pertinencia, a la capacidad de realizar parametrizaciones o derivaciones sucesivas: objeto, variables, dimensiones e indicadores para profundizar el conocer del objeto de la investigación.

Un modelo es la representación de procesos, relaciones de un conjunto de elementos con el objetivo de aprehender el conocimiento entorno a un objeto investigado o de estudio. Es aquello que se diseña para ser aprovechado como referente para generar a producción conocimientos o nuevas verdades. El modelo es un arquetipo orientador para docentes y estudiantes. Véase el siguiente modelo:

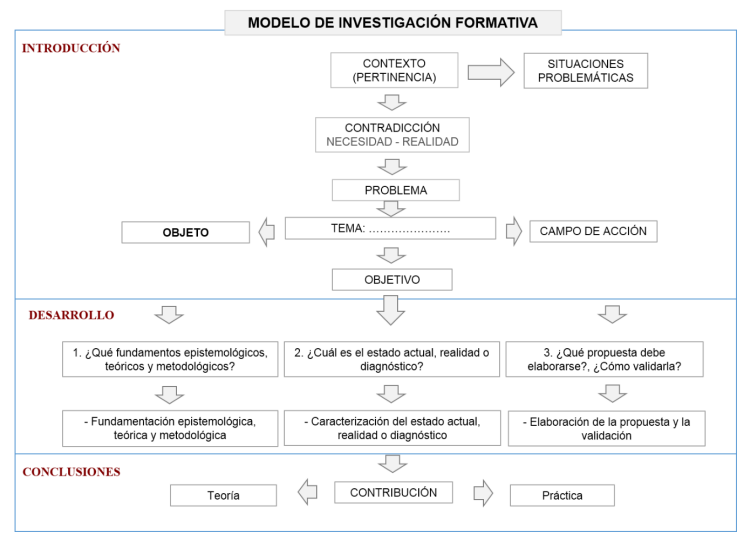

Figura 1: Modelo de investigación formativa Fuente: Elaboración propia

El método de la modelación tiene como resultado el modelo de investigación formativa para docentes y estudiantes de educación superior, con sus partes de Introducción, Desarrollo, Conclusiones, con sus componentes para profundizar el conocimiento.

Introducción: parte del estudio de pertinencia, en el que se debe precisar las necesidades y requerimientos del contexto, determinados en los planes de desarrollo nacional, zonal, regional o locales. Se deberá precisar las situaciones problemáticas, que lleve a establecer la contra- dicción entre realidad actual y la necesidad deseada acerca del problema.

El tema debe ser concreto, novedoso, relevante, pertinente y debe responder a un programa y a las líneas de investigación de la Institución y las sub líneas o ámbitos declarados por la Carrera. Una vez planteado el tema se debe precisar el objeto (¿Qué investigo?) con base en la fundamentación epistemológica y teórica. Y el campo de acción se refiere a aquella parte del objeto que transformo con base en la caracterización del estado actual, realidad o diagnóstico, determinado en un tiempo y en un espacio. El objetivo es la declaración del propósito o la finalidad que el investigador se plantea alcanzar.

Desarrollo: Para dar cumplimiento al objetivo se deben plantear tres preguntas investigativas con sus correspondientes tareas, que permitan: 1. La fundamentación epistemológica, teórica y metodológica; 2. Caracterización del estado actual, diagnóstico o realidad investigada; 3. Presentación de la propuesta o aporte que el investigador contribuye en el tema investigado. Esta propuesta tiene que ser validada.

El investigador por la profundidad y extensión de cada pregunta con su respectiva tarea puede organizar en capítulos: Capítulo 1: Fundamentación epistemológica, teórica y metodológica; Capítulo 2: Caracterización del estado actual, diagnóstico; Capítulo 3: Propuesta y validación.

Conclusiones: Se precisan los resultados o los logros alcanzados en el tratamiento del problema investigado; así como, incluir aseveraciones que demuestren que el objetivo propuesto se ha cumplido. Las argumentaciones de cada epígrafe deben ser lo más relevantes. También se debe indicar el aporte a la teoría investigada y a la práctica generada desde la propuesta. Las conclusiones se redactan mediante una serie de incisos.

El rigor académico de una investigación se alcanza con base en referencias textuales, con sus respectivas fuentes bibliográficas. Las normas de escritura estarán conforme a las exigencias de la Revista en la que va a realizar la publicación o de la Institución en el caso de trabajos de grado y posgrado.

El enfoque interdisciplinar en trabajos académicos e investigativos contribuyen a profundizar en el conocimiento, más aún cuando se motiva a que los investigadores, sean docentes o estudiantes, inicien con la respectiva fundamentación epistemológica del objeto investigado, la utilización de metodologías activas y el aprovechamiento de las tecnologías.

Modelo que se recomienda para realizar investigaciones dentro del aula de clase, con base en la orientación de los docentes y sus estudiantes.

A futuro se pretende presentar un proyecto investigativo que valide el modelo propuesto aplicado en diferentes áreas del conocimiento.

\section{Referencias Bibliográficas}

Álvarez, J., Grau, S., y Pertegal, M. (2016). El trabajo 
colaborativo y la pedagogía del contexto. Innovación metodológica, 74-96.

Baranda, S. (2005). La investigación y la Universidad en América Latina (Inf. Téc.).

Boff, L. (2002). El cuidado esencial: Ética de lo humano compasión por la Tierra. Madrid: Trotta.

Cárdenas, M., Guerra, C., y Soler, Y. (2017). Impacto social de la formaación de competencias investigativas mediadas por las TIC. Didáctica y Educación, 8(2), $1-20$.

Cotino, L. (2012). El derecho a la educación como derecho fundamental. . Madrid: Centro de estudios políticos y constitucionales.

De la Fe Rodríguez, C., Vidaurreta-Porrero, I., Gómez, A., y Corral, D. (2015). El método de estudio de casos: Una herramienta docente válida. Revista Electrónica Interuniversitaria de Formación del Profesorado,, 127-137.

Enciclopedia Universal Ilustrada Europeo Americana. (1930). Espasa Galpe. Barcelona: Hijos de Espasa.

Espinoza, E., Rivera, A., y Tinoco, N. (2016). Formación de competencias investigativas en los estudiantes universitarios. Atenas, 1(33), 18-31.

Gómez, F. (2010). El nuevo paradigma de la complejidad y la educación: una mirada histórica*. Polis, 183-198.

González-Pérez, E. (2015). Estudio de casos como estrategia didáctica en la formación del estudiantado en $\mathrm{Bi}$ bliotecología. Revista e-Ciencias de la Información, $1-14$.

Hurtado, J., y Rodríguez, E. (2016). Desarrollo de la competencia comunicativo-investigativa en la educación superior. Investigación con métodos mixtos y educación por competencias en Perú. Investigar con y para la sociedad, 669-680.

Morin, E., y Pakman, M. (1990). Introducción al pensamiento complejo. Barcelona: Gedisa.

Navarrete, Z. (2017). La investigación en educación. Revista Mexicana de Investigación Educativa, 22(72), 229-234.

Ortega, A., y Gasset, D. (1957). Obras inéditas: El hombre y la gente. Revista de occidente. Madrid: Ograma.

Piñera, H. (1959). Introducción a la filosofía. La Habana. Habana: Cultural.

Portela-Falgueras, R., Ortega, Y., y Amaro, L. (2018). Reflexiones sobre el componente investigativo en la formación del profesional: propuestas desde la Universidad Católica de Cuenca-Ecuador. Revista Referencia Pedagógica, 201-215.

Presta, F. (2015). Pensar la epistemología universitaria en el debate contemporáneo. OXÍMORA(6), 83-93.

Ramírez, D., y Santana, J. (2014). El aprendizaje basado en proyectos y el aprendizaje de conceptos de calor y temperatura mediante aplicaciones en cerámica. Innovación educativa, 52-55.

Reibán, R., Rodriguez, H., y Zeballos, J. (2017). Competencias investigativas en la Educación Superior.
Publicando, 1(10), 395-405.

Shakespeare, W., y Hamlet. (1998). $S$ (19 ed.). (L. Fernández de Moratín, Trans.) Santafé de Bogotá, Colombia: . Bogotá: Panamericana.

Tapia, E., Ledo, C., y Estrabao, A. (2017). Fundamentos epistemológicos de la investigación formativa en las universidades de Ecuador. Didase@lia: Didáctica y Educación, $\operatorname{VIII}(7), 1-13$.

Travieso, D., y Ortiz, T. (2018). Aprendizaje basado en problemas y enseñanza por proyectos: alternativas diferentes para enseñar. Revista Cubana de Educación Superior, 123-136.

Velandia, C., Serrano, P., y Martín, A. (2017). Formative Research in Ubiquitous and Virtual Environments in Higher Education/La investigación formativa en ambientes ubicuos y virtuales en Educación Superior. Comunicar, 9-18.

Vildósola, X. (2017). El conocimiento sobre la epistemología de la ciencia como eje para mejorar la relación investigación-práctica en la formación inicial docente en ciencias: El caso de Chile. Enseñanza de la ciencias(Extraordinario), 89-95.

Zeña, C. (2015). La epistemología en la investigación universitaria. UCV-HACER. Revista de Investigación y Cultura, 4(2), 122-127.

Recibido: 10 de septiembre de 2019

Aceptado: 15 de mayo de 2020 
